\title{
Study on the measurement of global sizes of cylindrical parts using Talyrond 585LT
}

\author{
Zexiang Zhao ${ }^{a^{*}}$, Xinyu Zhao ${ }^{\mathrm{b}}$, Ruyi Liu ${ }^{\mathrm{a}}$, Dongxu Ren ${ }^{\mathrm{a}}$, Yanlong Cao ${ }^{\mathrm{a}}$, Wenhan Zeng ${ }^{\mathrm{a}}$ \\ ${ }^{\mathrm{a}}$ School of Mechatronics Engineering, ${ }^{\mathrm{b}}$ School of Computer Science. \\ Zhongyuan University of Technology \\ Zhengzhou, China \\ zexiang_zhao@zut.edu.cn
}

\begin{abstract}
The measurement method of the global sizes of cylindrical parts based on Talyrond 585LT and the standard ring was proposed. The changing processes from the roundness profiles measured by Talyrond 585LT to the actual roundness profiles was studied for holes and shafts, respectively. The evaluation models of the least-squares diameter, minimum circumscribed diameter, maximum inscribed diameter and minimax diameter of cylindrical parts were given. The roundness profiles of two holes and two shafts were extracted and changed into their corresponding actual roundness profiles. Their global sizes were evaluated, and their measurement uncertainties were analyzed and estimated. The analysis and experiment results showed that the above measuring method can meet the measurement need of the global sizes of cylindrical parts for implementation of ISO $14405-1$ in the manufacturing industry.
\end{abstract}

Keywords-global sizes; least-squares diameter; minimum circumscribed diameter; maximum inscribed diameter; minimax diameter

\section{INTRODUCTION}

Cylindrical parts are the widely used parts of mechanical devices, and their standardization requirements in dimension and geometry are higher and higher for the development of digital and intelligent manufacturing fields. Cylindrical parts can be indicated their nominal sizes \& limit deviations and geometrical tolerances according to ISO 286-1[1] and ISO 1101[2], respectively. The above indications of cylindrical parts need to measure their actual sizes and geometrical errors by using the length measuring gauges/instruments and geometrical error measuring instruments, respectively. The geometrical tolerances of cylindrical parts include roundness tolerance, generatrix tolerance, axis straightness tolerance and cylindricity tolerance, and their corresponding geometrical errors are roundness error, generatrix error, axis straightness error and cylindricity error, which can be measured by using the cylindricity measuring instruments. However, the fit characteristics of hole and shaft are determined by the comprehensive dimensions of the mating internal and external cylindrical parts, which are formed by their actual sizes and geometrical errors. Therefore, the global sizes and calculated sizes of cylindrical parts were defined in ISO $14405-1$ by ISO/TC 213 . The global sizes of cylindrical part include least-squares diameter, minimum circumscribed diameter, maximum inscribed diameter and minimax diameter, and the calculated sizes include circumference diameter, area diameter and volume diameter[3]. In fact, volume diameter is also a calculated global size. The $1^{\text {st }}$ and $2^{\text {nd }}$ versions of ISO 14404-1 were published in 2010 and 2016, respectively. Now that the global sizes and calculated sizes have be defined in ISO 14405-1, which will be gradually accepted in mechanical industry and face their measurement challenges. According to their definitions, the cylindrical coordinate measuring machines $\left(\mathrm{C}^{2} \mathrm{M}^{2}\right)$ are their suitable measuring instruments. However, almost all the cylindricity or roundness measuring instruments are pseudo cylindrical coordinate measurement machines $\left(\mathrm{p}-\mathrm{C}^{2} \mathrm{M}^{2}\right)$, which can only measure the relative radial variations of the sampling points in the roundness profiles of cylindrical part, but the absolute radial distances from sampling points in the roundness profiles of cylindrical part to the rotary axis of the rotary table should be measured for the evaluation of the global sizes and calculated sizes of the cylindrical parts. At present, the measuring instruments of the global sizes and calculated sizes of the cylindrical part have not been seen in the market. Owing to that there are many cylindricity measuring instruments in mechanical enterprises, they can be used as the basic measuring instruments of global sizes and calculated sizes. Since the cylindricity measuring instruments are $\mathrm{p}-\mathrm{C}^{2} \mathrm{M}^{2}$, the issue on the measurement of the absolute radial distances between the sampling points in the roundness profiles and the rotary axis of the rotary table of the $\mathrm{p}-\mathrm{C}^{2} \mathrm{M}^{2}$ should be studied. In this paper, we take Taylor 585TL as an example to study the measurement and evaluation issues of global sizes and calculated sizes of cylindrical parts by using the $\mathrm{p}-\mathrm{C}^{2} \mathrm{M}^{2}$ for promoting the implementation of ISO 14405-1 in the manufacturing industry.

\section{INTRODUCTION OF TAYLOR 585LT}

Talyrond 585LT is a type of cylindricity measuring instrument, which are made in Taylor Hobson Ltd., Co. as shown in Figure 1. The geometrical errors of cylindrical parts, such as, roundness error, generatrix straightness error, axis straightness error, cylindricity error, flatness error, coaxality error, perpendicularity error and so on, can be measured by using the instrument, and its rotary table has the automatic centering and leveling functions. Its main technique parameters are as follows: (1) maximum measuring weight: $75 \mathrm{~kg}$; (2)maximum measuring height: 500mm; (3)maximum measuring diameter: $\varnothing 400 \mathrm{~mm}$; (4) radial limit error: $\pm(0.015 \mu \mathrm{m}+0.0003 H \mu \mathrm{m})$ under the condition that the cutoff frequency is 1150UPR(undulation per round), where $H$ is the distance 
from the sampling point to the top of the rotary table in $z$ direction and its unit is $\mathrm{mm}$; (5) maximum resolution:

$0.5 \mathrm{~nm}$; 6) maximum sampling points in a roundness profile: 18000. The sampling point coordinate in $z$ axis can be measured by using a grating displacement sensor on the vertical column. The size of sampling point in the radial direction can be obtained by using a grating displacement sensor and the inductance sensor on the movable horizontal arm, and the angle between the sampling point and $x$ axis can be measured by using a circular grating sensor. For the measurement of roundness error, axis straightness error, cylindricity error and coaxality error, the roundness profiles are extracted, as shown in Figure 2. There are four evaluation methods for roundness errors and cylindricity errors, such as least-squares method, minimum circumscribed method, maximum inscribed method and minimum zone method. We can obtain the $x, y$ and $z$ coordinates of the sampling points of the roundness profiles of a cylindrical part, and it should be noted that the $z$ axis and the rotary axis of the rotary table do not coincide and there exists a system error between two axes above. Therefore, we can't directly use the coordinates of sampling points of roundness profiles to evaluate global sizes and calculated sizes, and the system error above should be checked and eliminated.

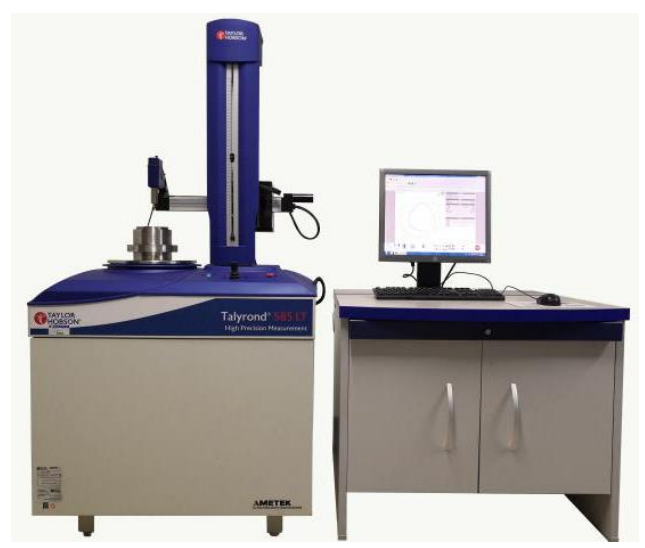

Figure 1. Talyrond 585LT

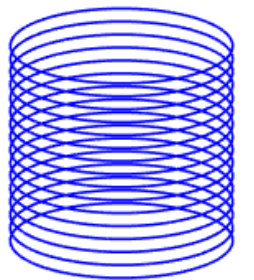

Figure 2. Roundness profiles

\section{COORDINATE TRANSFORMATION OF ROUNDNESS} PROFILE

\section{A. Coordinate Transformation from Cartesian} Coordinate System to Cylindrical Coordinate System

Suppose that the $x, y$ and $z$ coordinates of the $j$ th sampling point in the $i$ th roundness profile of a cylindrical part in xoyz Cartesian coordinate system, extracted by using Talyrond 585LT, are $x_{i j}, y_{i j}$ and $z_{i j}$, respectively. For the $j$ th sampling point in the $i$ th roundness profile, the relationship between its coordinates $x_{i j}, y_{i j}$ and $z_{i j}$ in Cartesian coordinate system and its corresponding coordinates $\rho_{i j}, \varphi_{i j}$ and $z_{i j}$ in cylindrical coordinate system, shown in Figure 3 , is as follows,

$$
\left\{\begin{array}{l}
\rho_{i j}=\sqrt{x_{i j}^{2}+y_{i j}^{2}} \\
\varphi_{i j}=\tan ^{-1} \frac{x_{i j}}{y_{i j}}=(j-1) \times \Delta \varphi, i=1 \sim m, j=1 \sim n \\
z_{i j}=z_{i j}, z_{i 1}=z_{i 2}=\cdots z_{i n}=z_{i}
\end{array}\right.
$$

where $n$ and $m$ are the sampling point number of a roundness profile and the number of roundness profiles of a cylindrical part, and $\Delta \varphi$ is the angle between two adjacent sampling points in a same roundness profile, which is equal to $2 \pi / n$.

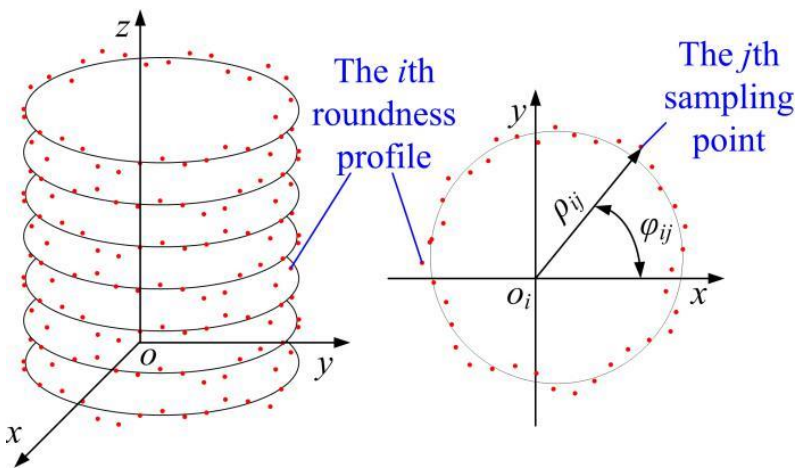

Figure 3. Implications of $\rho_{i j}$ and $\varphi_{i j}$

B. Determination of the System Error between $z$ Axis in Cylindrical Coordinate System and the Rotary Axis of Rotary Table

Owing to that $\rho_{i j}, i=1 \sim m, j=1 \sim n$, is not an absolute distance from the $j$ th sampling point in the $i$ th roundness profile of a cylindrical part to the rotary axis of the rotary table of Talyrond 585LT, the modification of $\rho_{i j}$ is needed through the determination of the system error between $z$ axis in cylindrical coordinate system and the rotary axis of the rotary table for gaining its corresponding actual distance from the sampling point to the rotary axis.

Before the measurement of cylindrical hole or shaft, the diameter of a standard ring should be first measured, and the $x$ and $y$ coordinates of the $j$ th sampling point of the ring are $x_{\mathrm{r} j}$ and $y_{\mathrm{r} j}$, respectively. Its absolute radial distance can be calculated as follows,

$$
\rho_{\mathrm{r} j}=\sqrt{x_{\mathrm{r} j}^{2}+y_{\mathrm{r} j}^{2}}, j=1 \sim n_{\mathrm{r}}
$$

where $n_{\mathrm{r}}$ is the sampling number of the ring's profile, as shown in Figure 4, which can be equal to $n$, and the diameter of the least-squares circle of the ring is as follows,

$$
\bar{R}_{\mathrm{r}}=\frac{1}{n_{\mathrm{r}}} \sum_{j=1}^{n_{\mathrm{r}}} \rho_{\mathrm{r} j}
$$

Since $\rho_{\mathrm{r} j}$ is not the actual distance from the $j$ th sampling point of the standard ring to the rotary axis of the rotary table, for reducing the calibration error, $\rho_{\mathrm{r} j}$ of the $j$ th sampling point of the ring should be modified as follows,

$$
\rho_{\mathrm{c} j}=D_{\mathrm{r}} / 2+\left(\rho_{\mathrm{r} j}-\bar{R}_{\mathrm{r}}\right), j=1 \sim n_{\mathrm{r}}
$$

where $D_{\mathrm{r}}$ may be the actual size or the mean of the actual sizes or the actual diameter of the least-squares circle 
gained through calibration. Based on the values of $\rho_{\mathrm{c} j}$, $j=1 \sim n_{\mathrm{r}}$, the center coordinates $a_{\mathrm{c} 0}$ and $b_{\mathrm{c} 0}$ of the ring or the ring's least-squares circle can be determined as follows,

$$
\left\{\begin{array}{l}
a_{\mathrm{c} 0}=\frac{1}{n_{\mathrm{r}}} \sum_{j=1}^{n_{\mathrm{r}}} \rho_{\mathrm{c} j} \cos \left((j-1) \times \frac{2 \pi}{n_{\mathrm{r}}}\right) \\
b_{\mathrm{c} 0}=\frac{1}{n_{\mathrm{r}}} \sum_{j=1}^{n_{\mathrm{r}}} \rho_{\mathrm{c} j} \sin \left((j-1) \times \frac{2 \pi}{n_{\mathrm{r}}}\right)
\end{array}\right.
$$

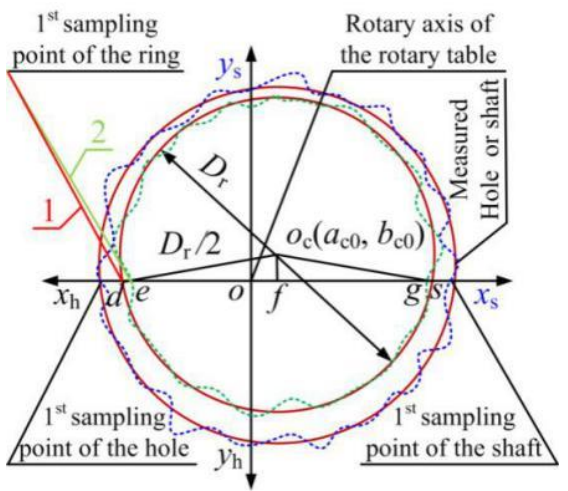

Figure 4. Schematic of relationship between the measured hole or shaft and the calibration ring

It is known that the actual radial distance from the $1^{\text {st }}$ sampling point of the ring to the rotary axis of the rotary table is $\rho_{\mathrm{c} 1}$. If the actual size or the actual mean of the ring is used to determine the system error between $z$ axis and the rotary axis of the rotary table, we think that the profile of the ring is an ideal circle, as shown in Figure 4, and $\rho_{\mathrm{c} 1}$, is equal to the distance $L_{d o}$ from the $1^{\text {st }}$ sampling point $d$ to the rotary center $o$, which can be calculated as follows,

$$
L_{d o}=\sqrt{\left(\frac{D_{\mathrm{r}}}{2}\right)^{2}-b_{\mathrm{c} 0}^{2}}+a_{\mathrm{c} 0}
$$

The system error between $z$ axis and the rotary axis of the rotary table can be calculated as follows,

$$
\Delta_{\mathrm{c} 1}=\rho_{\mathrm{c} 1}-L_{d o}
$$

If the profile of the ring is not an ideal circle, $D_{\mathrm{r}}$ is the diameter of the least-squares circle of the profile, the system error between $z$ axis and the rotary axis of the rotary table can be calculated as follows,

$$
\Delta_{\mathrm{c} 1}=\rho_{\mathrm{c} 1}-\left(L_{d o}+\left(\rho_{\mathrm{r} 1}-\bar{R}_{\mathrm{r}}\right)\right)
$$

After determination of the system error above, if the hole is measured, its $\rho_{i j}$ calculated by(1) can be transferred into the actual radial distance from the $j$ th sampling point in the $i$ th roundness profile to the rotary axis of the rotary table as follows,

$$
\rho_{\mathrm{h} i j}=\rho_{i j}-\Delta_{\mathrm{c} 1}, i=1 \sim m, j=1 \sim n
$$

If the shaft is measured, the system error above can be calculated by (10) and (11), where $D_{\mathrm{r}}$ is the actual size or the mean of the actual sizes and the least-squares diameter of the ring's profile, respectively.

$$
\Delta_{\mathrm{c} 2}=\rho_{\mathrm{c} 1+} \rho_{\mathrm{c}(n / 2+1)}-2 \sqrt{\left(\frac{D_{\mathrm{r}}}{2}\right)^{2}-b_{\mathrm{c} 0}^{2}}
$$

$$
\Delta_{\mathrm{c} 2}=\rho_{\mathrm{c} 1}+\rho_{\mathrm{c}(n / 2+1)}-\left[\begin{array}{l}
2 \sqrt{\left(\frac{D_{\mathrm{r}}}{2}\right)^{2}-b_{\mathrm{c} 0}^{2}}+\left(\rho_{\mathrm{r} 1}-\bar{R}_{\mathrm{r}}\right) \\
+\left(\rho_{\mathrm{r}(n / 2+1)}-\bar{R}_{\mathrm{r}}\right)
\end{array}\right]
$$

For the measured shaft, its $\rho_{i j}$ calculated by (1) can be transferred into the actual radial distance from the $j$ th sampling point in the $i$ th roundness profile to the rotary axis of the rotary table as follows,

$$
\rho_{\mathrm{s} i j}=\rho_{i j}-\Delta_{\mathrm{c} 2}, i=1 \sim m, j=1 \sim n
$$

\section{EVALUATION MODELS OF GLOBAL SIZES OF CYLINDRICAL PARTS}

As mentioned above, for cylindrical parts, there are four kinds of global sizes, that is, least-squares diameter, minimum circumscribed diameter, maximum inscribed diameter and minimax diameter, as shown in Figure 5.

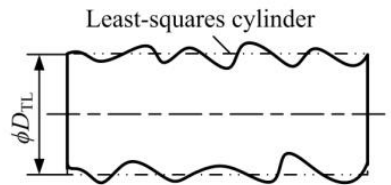

(a) Least-squares

Maximum inscribed cylinder

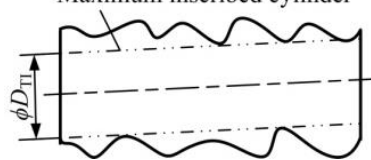

(c) Maximum inscribed Figure 5. Schematic of four kinds of global sizes

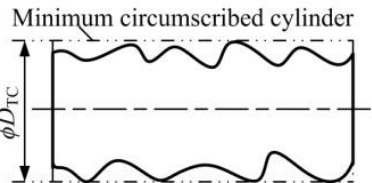

(b) Minimum circumscribed Minimax cylinde

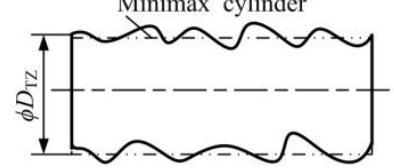

(d) Minimax
The evaluation model of least-squares diameter can be built based on the actual radial sizes of the cylindrical roundness profiles calculated by (9) for a hole and by (12) for a shaft. In fact, the evaluation modeling of least-squares diameter is a nonlinear issue, but owing to that the centering and leveling of the measured part should be done before measurement of roundness profiles, the eccentricity and tilt angle of the axis of the measured part are less. Therefore, the non-linear least-squares issue above can be transferred into a linear least-squares one, which can be proceeded through solving linear equations. The least-squares diameter of a cylindrical part can be calculated as follows[4],

$$
D_{\mathrm{TL}}=\frac{2}{m n} \sum_{i=1}^{m} \sum_{j=1}^{n} \rho_{\mathrm{T} i j}
$$

where the subscript $\mathrm{T}$ can be replaced by $\mathrm{h}$ and $\mathrm{s}$ for hole and shaft, respectively.

According to the definitions of minimum circumscribed diameter and maximum inscribed diameter in Reference [5], the evaluation models of two kinds of global sizes of cylindrical part can be built as follows,

$$
\left\{\begin{array}{l}
D_{\mathrm{TC}}=2 \min _{X_{\mathrm{TC}}} \max _{\substack{1 \leq j \leq n \\
1 \leq i \leq m}} d_{\mathrm{TC} i j} \\
D_{\mathrm{TI}}=-2 \min _{X_{\mathrm{TI}}} \max _{\substack{1 \leq j \leq n \\
1 \leq i \leq m}}\left(-d_{\mathrm{TI} i j}\right)
\end{array}\right.
$$


where the meaning of T can be seen in (13), $X_{\mathrm{TC}}$ and $X_{\mathrm{TI}}$ are the axis parameter column vector of the minimum circumscribed reference cylindrical surface and the maximum inscribed reference cylindrical surface, respectively, where $X_{\mathrm{TC}}=\left[\begin{array}{lll}x_{\mathrm{TC} 0} & y_{\mathrm{TC} 0} & p_{\mathrm{TC}} q_{\mathrm{TC}}\end{array}\right]^{\mathrm{T}}$ and $X_{\mathrm{TI}}=\left[x_{\mathrm{TI}}\right.$ $\left.y_{\mathrm{TI} 0} p_{\mathrm{TI}} q_{\mathrm{TI}}\right]^{\mathrm{T}}, x_{\mathrm{TC} 0}$ and $x_{\mathrm{TI} 0}$ are the $x$ coordinates of one end of the axis of the reference cylindrical surfaces above in xoy plane, respectively, $y_{\mathrm{TC} 0}$ and $y_{\mathrm{TI} 0}$ are the $y$ coordinates of one end of the axis of the reference cylindrical surfaces above in xoy plane, respectively, $p_{\mathrm{TC}}$ and $p_{\mathrm{TI}}$ are equal to $\cos \alpha_{\mathrm{TC}} / \cos \gamma_{\mathrm{TC}}$ and $\cos \alpha_{\mathrm{TI}} / \cos \gamma_{\mathrm{TI}}$, respectively, $q_{\mathrm{TC}}$ and $q_{\mathrm{TI}}$ are equal to $\cos \beta_{\mathrm{TC}} / \cos \gamma_{\mathrm{TC}}$ and $\cos \beta_{\mathrm{TI}} / \cos \gamma_{\mathrm{TI}}$, respectively, where $\alpha_{\mathrm{TC}}, \beta_{\mathrm{TC}}$ and $\gamma_{\mathrm{TC}}$ are the angles between the axis of minimum circumscribed reference cylindrical surface and $x, y$ and $z$ axes, respectively, and $\alpha_{\mathrm{TI}}, \beta_{\mathrm{TI}}$ and $\gamma_{\mathrm{TI}}$ are the angles between the axis of maximum inscribed reference cylindrical surface and $x, y$ and $z$ axes, respectively. $d_{\mathrm{TU} i j}$ is the distance of the $j$ th sampling point in the $i$ th actual roundness profile to the axis of the corresponding reference cylindrical surface, which can be calculated as follows,

$d_{\mathrm{TU} i j}=\left[\begin{array}{l}\left(\rho_{\mathrm{T} i j} \cos \varphi_{\mathrm{T} i j}-x_{\mathrm{TU} 0}\right)^{2}+\left(\rho_{\mathrm{T} i j} \sin \varphi_{\mathrm{T} i j}-y_{\mathrm{TU} 0}\right)^{2}+z_{\mathrm{T} i j}^{2}+ \\ \frac{\left(\rho_{\mathrm{T} i j} \cos \varphi_{\mathrm{T} i j}-x_{\mathrm{TU} 0}\right)+\left(\rho_{\mathrm{T} i j} \sin \varphi_{\mathrm{T} i j}-y_{\mathrm{TU} 0}\right)+z_{\mathrm{T} i j}}{1+p_{\mathrm{TU}}^{2}+q_{\mathrm{TU}}^{2}}\end{array}\right]^{1 / 2}$

where the meaning of subscript $\mathrm{T}$ can be seen in (14) and the subscript $\mathrm{U}$ can be replaced by $\mathrm{C}$, I and $\mathrm{Z}$, which means that the parameters in (15) belong to minimum circumscribed reference cylindrical surface, maximum inscribed reference cylindrical surface and minimax reference cylindrical surface, respectively.

For the minimax diameter of cylindrical part, its evaluation model can be written as follows,

$$
D_{\mathrm{TZ}}=\max _{\substack{1 \leq j \leq n \\ 1 \leq i \leq m}} d_{\mathrm{TU} i j}+\min _{\substack{1 \leq j \leq n \\ 1 \leq i \leq m}} d_{\mathrm{TU} i j}
$$

where the following condition should be satisfied.

$$
f_{\mathrm{TZ}}=\min _{X_{\mathrm{TZ}}}\left\{\max _{\substack{1 \leq j \leq n \\ 1 \leq i \leq m}} d_{\mathrm{TU} i j}-\min _{\substack{1 \leq j \leq n \\ 1 \leq i \leq m}} d_{\mathrm{TU} i j}\right\}
$$

where $X_{\mathrm{TZ}}=\left[\begin{array}{lll}x_{\mathrm{TZ} 0} & y_{\mathrm{TZ} 0} & p_{\mathrm{TZ}} \\ q_{\mathrm{TZ}}\end{array}\right]^{\mathrm{T}}$, the meaning of which can be seen in (14).

From (15) and (16), the evaluation of minimum circumscribed diameter, maximum inscribed diameter and minimax diameter are actually the minimax issues, which can use optimization method to solve these problems. Three global sizes above correspond to minimum circumscribed method, maximum inscribed method and minimum zone method in the evaluation of cylindricity errors, respectively. There have been many optimization algorithms used for the evaluation of cylindricity errors by many researchers, such as, genetic algorithm[6], geometry optimization searching algorithm[7], kinematic geometry optimization algorithm[8] and so on[9,10]. These optimization algorithms can also be used for the evaluation of the global sizes.

\section{EXPERIMENTS AND DISCUSSION}

\section{A. Experiments}

Two holes and two shafts were measured by using Talyrond 585LT. Their nominal sizes $D_{\mathrm{N}}$ are $51 \mathrm{~mm}$, the numbers $m$ of roundness profiles of holes and shafts are 21 and 25 , respectively, and their corresponding measured lengths $L$ are $100 \mathrm{~mm}$ and $120 \mathrm{~mm}$, respectively, where $\Delta z$ is $5 \mathrm{~mm}$ for holes and shafts. The sampling number $n$ of a roundness profile is 18000 , and UPR is $1-500$, where UPR is the abbreviations of undulations per round. The standard ring was made in Mutitoto of Japan, the calibrated size $D_{\mathrm{r}}$ is $44.955 \mathrm{~mm}$. In this paper, we think this calibrated size as the diameter of its least-squares circle, and its sampling number and filter are 18000 and 1-500UPR, respectively. The ring's picture is shown in Figure 6(a), and its profile measured by using Talyrond 585LT can be seen in Figure $6(b)$, the local magnification of which is 2000 .

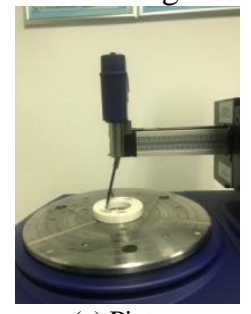

(a) Picture

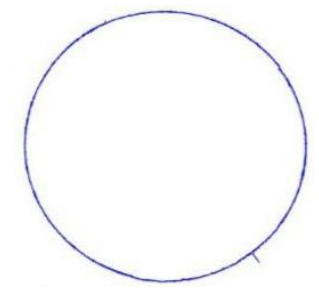

(b) roundness profile
Figure 6 Standard ring

The roundness profiles of two holes and two shafts are shown in Figure 7. Through a series of data processes for the roundness profiles in Figure 7 based on (1)-(12), their corresponding actual roundness profiles were obtained, and their least-squares diameter $(L S D)$, minimum circumscribed diameter $(M C D)$, maximum inscribed diameter $(M I D)$ and minimax diameter $(M M D)$ were evaluated based on (13), (14) and (16), where the optimization function 'fminimax' in Matlab was adopted. Their evaluation results are shown in TABLE I.

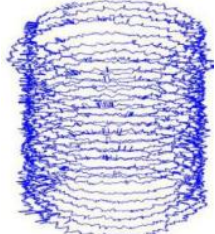

(a) Hole 1

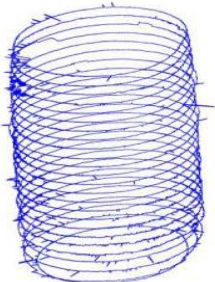

(c) Shaft 1

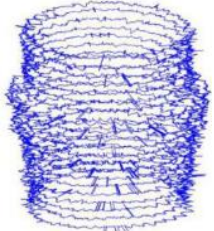

(b) Hole 2

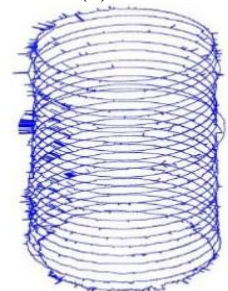

(d) Shaft 2
Figure 7. Roundness profile of the measured holes and shafts(local magnification :1000)

TABLE I. EVALUATION RESULTS OF GLOBAL SIZES

\begin{tabular}{|c|c|c|c|c|}
\hline \multirow{2}{*}{ Parts } & \multicolumn{4}{|c|}{ Global sizes/mm } \\
\cline { 2 - 5 } & $\boldsymbol{L S D}$ & $\boldsymbol{M C D}$ & $\boldsymbol{M I D}$ & $\boldsymbol{M} \boldsymbol{D D}$ \\
\hline Hole 1 & 51.0094 & 51.0018 & 50.9763 & 50.9889 \\
\hline
\end{tabular}




\begin{tabular}{|c|c|c|c|c|}
\hline \multirow{2}{*}{ Parts } & \multicolumn{4}{|c|}{ Global sizes/mm } \\
\cline { 2 - 5 } & $\boldsymbol{L S D}$ & $\boldsymbol{M C D}$ & $\boldsymbol{M I D}$ & $\boldsymbol{M M D}$ \\
\hline Hole 2 & 50.9948 & 51.0138 & 50.9771 & 50.9958 \\
\hline Shaft 1 & 51.0574 & 51.0717 & 51.0487 & 51.0608 \\
\hline Shaft 2 & 51.0607 & 51.0761 & 51.0545 & 51.0642 \\
\hline
\end{tabular}

\section{B. Discussion}

Even though we can transfer the roundness profiles measured by Talyrond 585LT into their corresponding actual roundness profiles of a part through the roundness profile of the standard ring and the data process in Section III, which can be used for the evaluation of its global sizes, it is should be noted that the measurement conditions of the part and the standard ring must be same, that is, their $x$ coordinate systems must be same. The equations in Section III are only adapted to the following conditions: $\rho_{\mathrm{r} 1}>D_{\mathrm{r}} / 2$, $D_{\mathrm{N}}>D_{\mathrm{r}}, \rho_{11}>\rho_{\mathrm{r} 1}$. Otherwise, $\Delta_{\mathrm{c} 1}=-\Delta_{\mathrm{c} 1}$. If $D_{\mathrm{N}}>D_{\mathrm{r}}$ and $\rho_{11}<\rho_{\mathrm{r} 1}$, $\rho_{i j}=\rho_{i j}+2\left(\rho_{11}-\rho_{\mathrm{r} 1}\right)$ for shaft, $\rho_{i j}=\rho_{i j}-2\left(\rho_{11}-\rho_{\mathrm{r} 1}\right)$ for hole.

Through the analysis of the evaluation models (13), (14) and (16) of the global sizes, the radial sizes $\rho_{i j}, i=1 \sim m$, $j=1 \sim n$, are the largest influence factors of their measurement uncertainties. The radial sizes' uncertainties may be influenced by many factors, such as the calibration uncertainty of the standard ring, the measuring uncertainties of the inductive displacement sensor and the grating sensor of the horizontal arm, the straightness error of vertical slide-way, the parallelism error of the rotary axis of the rotary table to the vertical slide-way, the rotary precision of the rotary table and so on. Through analyzing of the above factors, the calibration uncertainty of the standard ring is the main component of the radial size's uncertainty, which is $\pm 0.010 \mathrm{~mm}$. Therefore, the estimated measurement uncertainty of the global sizes may be less than $\pm 0.012 \mathrm{~mm}$. For improving the measurement precision of global sizes, the manufacturing and measurement precision of the standard ring or the standard cylinder must be higher. The measurement method of the global sizes is also adapted to other cylindricity measuring instruments.

\section{CONCLUSIONS}

The measurement method of the global sizes of cylindrical parts was proposed based on the roundness profiles' extraction by using Talyrond 585LT and their modification by using the standard ring. The changing processes from the roundness profiles to the actual roundness profiles were introduced for holes and shafts, respectively, and the evaluation models of the global sizes of cylindrical parts were built. The roundness profiles of two holes and two shafts were extracted and changed into their corresponding actual roundness profiles. Their leastsquares diameter, minimum circumscribed diameter, maximum inscribed diameter and minimax diameter were evaluated, and the latter three global sizes were obtained through optimization by using 'fminimax' function. The estimated measurement uncertainties of the above global sizes are about $\pm 0.012 \mathrm{~mm}$, which are mainly influenced by the calibration uncertainty of the standard ring. The above measuring method of the global sizes of cylindrical parts can be one of a variety of measurement methods in the future, which may facilitate the implementation of ISO $14405-1$ in the manufacturing industry.

\section{ACKNOWLEDGMENT}

The work was supported by the project of National Natural Science Foundation of China (Contract No.: 51475485).

\section{REFERENCES}

[1] ISO/TC 213, ISO 286-1:2010 Geometrical Product Specifications(GPS) - ISO code system for tolerances on linear sizes - Part 1:Basis of tolerances, deviations and fits, Switzerland, 2010

[2] ISO/TC 213, ISO 1101:2017 Geometrical product specifications(GPS) - Geometrical tolerancing - Tolerances of form, orientation, location and run-out, Switzerland, 2017

[3] ISO/TC 213, ISO 14405-1:2016 Geometrical product specifications (GPS) - Dimensional tolerancing -Part 1: Linear sizes, Switzerland, 2016

[4] Zexiang Zhao, Bin Li, Guoqing Zhang, Hechun Yu, Wenbo Wang, Xin He, Xiaoshuai Hou, Xupeng Cheng, Bo Yao, "Study on the evaluation of cylinder's global sizes", Precision Engineering, 2017, Vol. 49, pp. 189-199.

[5] Zexiang Zhao, Bin Li, Guoqing Zhang, Hechun Yu, Mengjiao Shang, "Inflfluence of eccentricity and tilt of cylindrical part's axis on the measurement results of its diameters",Measurement, 2019, Vol. 138, pp. 232-239.

[6] Hsin-Yi Lai, Wen-Yuh Jywe, Cha'o-Kuang Chen, ChienHong Liu,"Precision modeling of form errors for cylindricity evaluation using genetic algorithms", Precision Engineering, 2000, Vol. 24, pp. 310-319.

[7] Xianqing Lei, Hongwei Song, Yujun Xue, Jishun Li, Jia Zhou, Mingde Duan,"Method for cylindricity error evaluation using Geometry Optimization Searching Algorithm", Measurement, 2011, Vol. 44, pp. 1556-1563.

[8] Peng Zheng, Dongliang Liu, Fengxia Zhao, Linna Zhang, "An efficient method for minimum zone cylindricity error evaluation using kinematic geometry optimization algorithm", Measurement, 2019, Vol. 135, pp. 886-895.

[9] Adamczak S, Janecki D, Stępień K, "Qualitative and quantitative evaluation of the accuracy of the V-block method of cylindricity measurements", Precision Engineering, 2010, Vol. 34, pp. 619-626.

[10] Jian Mao, Yanlong Cao,Jiangxin Yang, “Implementation uncertainty evaluation of cylindricity errors based on geometrical product specification (GPS)", Measurement, 2009, Vol. 42, pp. 742-747. 